\title{
Trends in obesity at the national and local level among South Korean adolescents
}

\author{
Kyungsoo Han, ${ }^{1}$ Sejin Park, Jürgen Symanzik, ${ }^{2}$ Sookhee Choi, ${ }^{3}$ Jeongyong Ahn' \\ 'Department of Statistics, Chonbuk National University, Jeonbuk, Korea; \\ ${ }^{2}$ Department of Mathematics and Statistics, Utah State University, Logan, UT, USA; \\ ${ }^{3}$ Woosuk University, Jeonbuk, Korea
}

\begin{abstract}
Obesity is a global phenomenon affecting all socioeconomic groups, irrespective of age, gender or ethnicity. In many countries, obesity trends are causing serious public health concerns threatening the viability of basic health care delivery. In this article, we examine the trends of adolescent obesity at the national level in South Korea introducing a new approach for visualising data at the local level based on linked micromap plots. Our analysis shows that the obesity rates for 2013 have only increased slightly since 2006 for South Korean adolescents of both genders in various age groups. However, considerable increases could be observed for the subgroup of adolescent males and adolescent females living in rural areas. Trends at the local level show a slight increase of the prevalence of obesity in most regions of the country, with the highest obesity prevalence found in the Northeast.
\end{abstract}

Correspondence: Jeongyong Ahn, Department of Statistics, Institute of Applied Statistics, Chonbuk National University, 561-756 Jeonbuk, Korea. Tel: +82.63 .2703392 .

E-mail: jyahn@jbnu.ac.kr

Key words: Data visualisation; Geographic patterns; Linked micromap plots; Obesity; South Korea.

Acknowledgments: this paper was supported by research funds of Chonbuk National University in 2012. This research was also supported by the Basic Science Research Program through the National Research Foundation (NRF) of Korea funded by the Ministry of Education (NRF2012R1A1A4A01002729).

Received for publication: 29 May 2015.

Revision received: 15 September 2015.

Accepted for publication: 10 December 2015.

(C) Copyright K. Han et al., 2016

Licensee PAGEPress, Italy

Geospatial Health 2016; 11:381

doi:10.4081/gh.2016.381

This article is distributed under the terms of the Creative Commons Attribution Noncommercial License (CC BY-NC 4.0) which permits any noncommercial use, distribution, and reproduction in any medium, provided the original author(s) and source are credited.

\section{Introduction}

The prevalence of obesity among children and adolescents has become a serious epidemic health problem that is now estimated to be the fifth leading cause of mortality in the world (James et al., 2008). Excess body weight can also affect the quality of life, education and income potential (Kolotkin et al., 2001; McLaren, 2007; Puhl and Heuer, 2009). In addition, obesity is also associated with various diseases and it increases the risk of premature death (Fontaine et al., 2003; Pichainarong et al., 2006). Analysing the data and reporting the statistics are therefore important for the identification of health trends and establishment of normal health patterns. Overweight and obesity are most likely the result of complex interactions between genes, lifestyles, dietary habits and socioeconomic factors. As the targets of many public health strategies, life-related factors are modifiable and have been highlighted in many investigations. It is evident that life-related factors, such as physical activity and eating habits, are associated with paediatric overweight and obesity (Han et al., 2010).

In many cases, health data are presented in extensive data tables that are often sorted alphabetically. However, such tabular presentations tend to be uninformative as it is difficult to quickly find the extreme values (minimum and maximum) in a table and to identify table rows with similar contents. In case of geographic information, tables usually do not allow for a quick link between table rows representing specific geographic regions and the locations of these regions on a map. This fact suggests that the conversion of tabular data into a visual context would better illustrate patterns and relationships in the data as pointed out by Gebreab et al. (2008). In addition, interpreting and understanding health data usually requires the consideration of the appropriate spatial context because many health datasets come with geographic information. Some health studies in the past incorporated the spatial context in the data analysis (Edsall, 2003; Ezzati et al., 2006; Jacob et al., 2010). Most of the past studies, however, used only simple images and maps, such as aerial images, choropleth or isopleth maps for the visualisation of health data. Such images and maps have been popular in spite of several problems and limitations (Dent, 1993; Harris, 1999). The greatest problem is that it is difficult to show more than one statistical variable in an aerial image or a map. Another problem is that a viewer cannot easily observe trends, relationships and anomalies that may be present (Gebreab et al., 2008). To solve these problems, we use linked micromap (LM) plots, a statistical graph that combines multiple small maps and statistical data panels. LM plots were first introduced in the United States (USA) in 1996 by Carr and Pierson (1996) and have been used for various medical and public health applications in the past (Carr, 2001; Wang et al., 2002). Comprehensive overviews for the use and creation of LM plots have been published by Symanzik and Carr (2008) and Carr and Pickle (2010). LM plots are graphs that link statistical information to an 
ordered set of small maps in order to easily explore and communicate patterns in the outcome variable, explanatory variables, geographic locations and the associations among them. LM plots make use of aggregated values, so each individual's information is kept strictly confidential. In fact, LM plots are not designed to show specific data at a particular geographic location but, rather, to group information into meaningful geographical subregions, such as states, provinces, or districts. LM plots have been adapted for Korea only recently (Ahn, 2013; Han et al., 2014). Although they have been used for a variety of other medical and health data sets in the United States (Carr, 2001; Gebreab et al., 2008), to the best of our knowledge, they have not been used in the obesity context. In this study, we describe how spatial patterns of the prevalence of obesity and life-related indicators of adolescents in South Korea can be examined visually and simultaneously using LM plots. First, we examine the trends of adolescent obesity at the national level comparing the rates of obesity and other indicators in the two groups, metropolitan versus rural and male versus female. Second, we visualise the obesity data at the local level using LM plots, with which we can better explore and understand the relationships of the variables and geographic patterns, which are inherent in the data.

\section{Materials and Methods}

\section{Data sources}

To analyse and visualise the prevalence of obesity of adolescent in Korea, we used the Korean Youth Risk Behavior Web-based Survey (KYRBWS) (http://www.yhs.cdc.go.kr) data for the period from 2006 to 2013. The data were obtained from the Korean Statistical Information Service (KOSIS) (http://www.kosis.kr). KOSIS is the gateway for Korea's official statistics and provides access to data from more than 120 statistical agencies covering more than 500 different topics. We aggregated the data into the 16 upper-level, local autonomies of Korea using the traditional geographic breakdown prior to the creation of Sejong as a special autonomous city in 2007 . As of 2006 , Korea is divided into eight provinces, one special autonomous province, six metropolitan cities and one special autonomous city. The dataset used in this article was obtained from KOSIS and it consists of eleven variables and a region name. Table 1 lists eight indicator variables and three demographic variables from the data set used in this study.

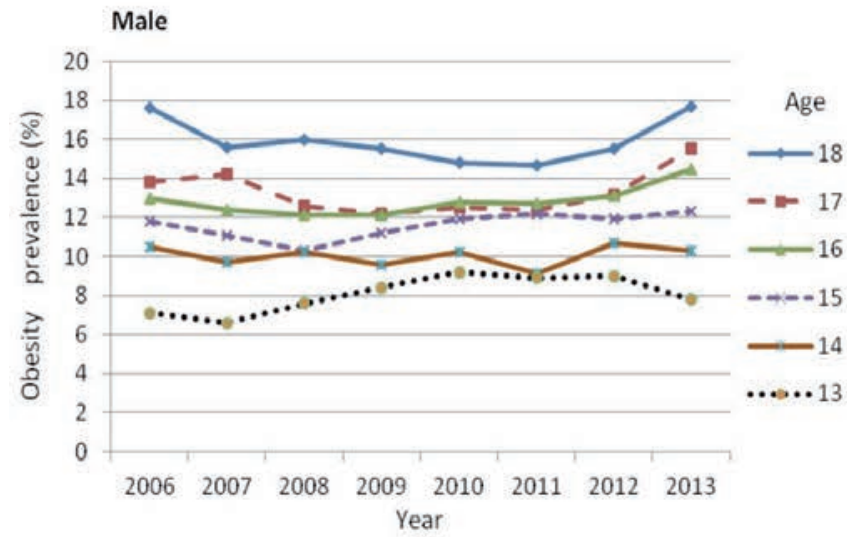

Metropolitan areas often provide better socioeconomic conditions (such as educational level, income, and wealth) than rural areas. Therefore, we divided the 16 upper-level local autonomies of Korea into two groups, metropolitan (Seoul, Busan, Daegu, Incheon, Gwangju, Daejeon, Ulsan, and Gyeonggi) and rural (Gangwon, Chungbuk, Chungnam, Jeonbuk, Jeonnam, Gyeongbuk, Gyeongnam, and Jeju), according to the extent of urbanisation to determine whether the means of these two groups are equal.

\section{Visualisation}

To visualise the geographically referenced data, LM plots use three or more sequence panels (micromap panel, label panel and one or more statistical summary panels) linked in parallel by location. The micromap panel contains miniature drawings outlining a region. This makes the shape and neighbourhood relationships of small geographic

Table 1. Variables from the Korean Statistical Information Service data set.

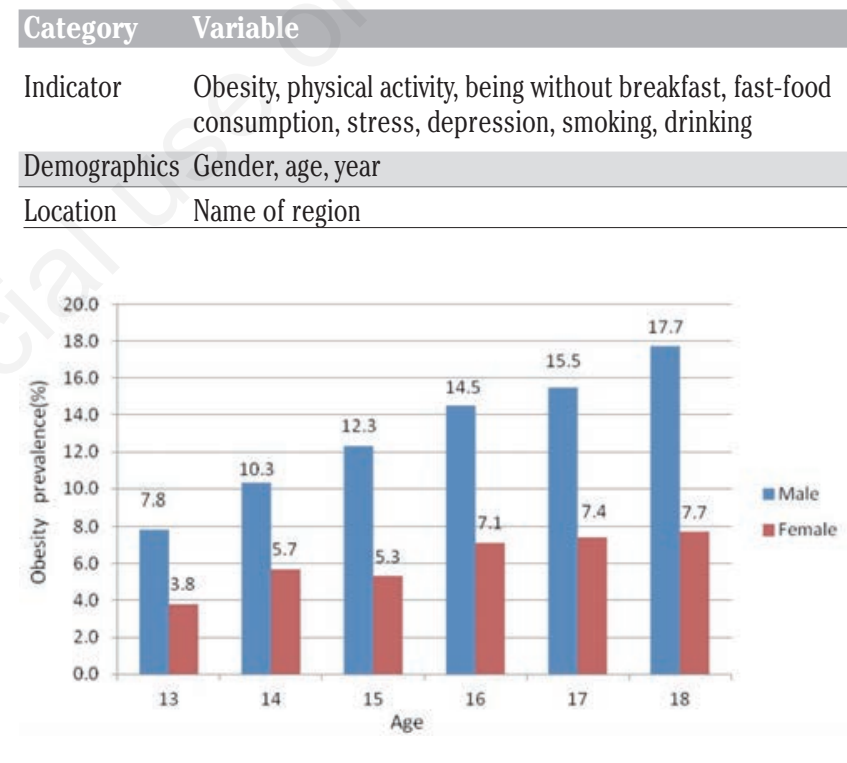

Figure 1. The prevalence of obesity by age and gender in 2013.

Figure 2. Trends of the prevalence of obesity by year, gender and age.

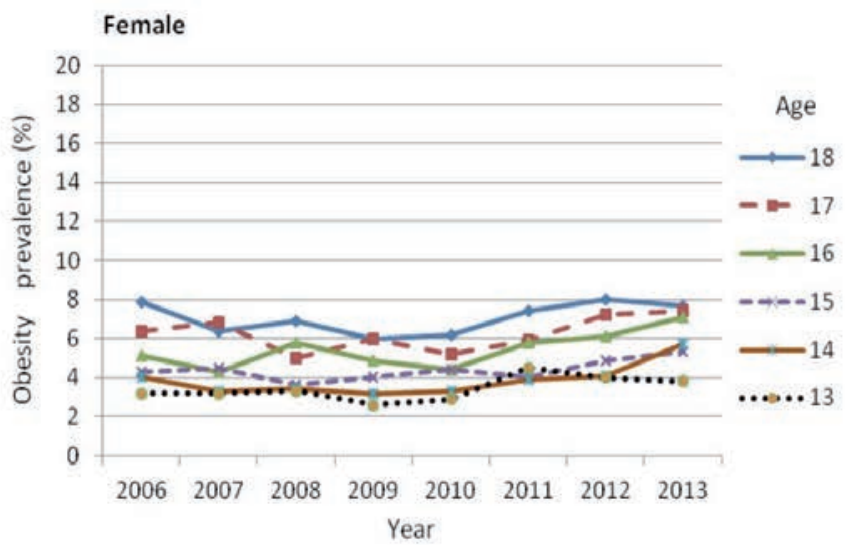


subregions more visible while still maintaining the relevant information in the miniature maps. The label panel gives the names of the geographic subregions, while the statistical panels can represent many different forms of statistical summaries, including box-plots, dot-plots, time series plots, confidence intervals (CI) and others. In addition, the geographic subregions in LM plots are sorted based on the statistical variable of interest, which improves perception among consecutive panels from the top to the bottom of the display. To draw the viewer's attention to focus on a specific area at a time, LM plots divide the map regions into perceptual groups of five or fewer subregions that are highlighted on one of the small maps. The geographic locations, the names of the subregions and the statistical data are linked via a colour scheme. These features make it possible to visualise specific geographic patterns in the data that are often lost in other types of graphs and maps. A good explanatory example of a LM plot that further describes the overall concept can be found in Gebreab et al. (2008).

\section{Statistical analysis}

To explore and analyse the data, common data analysis methods were used in this study. Exploratory data analysis methods are primarily used to explore the data before using more quantitative traditional methods. We used two-sample t-tests (Snedecor and Cochran, 1989) to compare the means of the variables in the two groups. In addition, we fitted regression models to statistically investigate the temporal trends of the prevalence of obesity. With such models, we could determine the relationship between obesity rate and time. Lastly, rank correlation analysis was used to measure the relationship between obesity and other indicators.

\section{Results}

\section{Data analysis at the national level}

Figure 1 shows the prevalence of obesity for 2013 by age and gender. The prevalence of obesity for adolescent males in each age group is about twice as high compared to that for adolescent females. The causes for this difference are not immediately obvious. However, an examination of studies with attention to potential gender differences reveals that such differences are common, both before and during puberty (Reilly and Wilson, 2006; Wisniewski and Chernausek, 2009). Moreover, the prevalence of obesity for both genders at age 18 is about twice as high as the prevalence of obesity at age 13 .

Figure 2 shows the temporal development of the obesity rate by gender and age group from 2006 to 2013 . There were no statistically significant trends in any of the six age groups (13 to 18) for any of the genders. However, a slightly increasing trend from 2006 to 2013 was observed for ten of the twelve age/gender combinations. Only the obesity prevalence of adolescent males at the age of 14 , and of adolescent females at the age of 18, decreased slightly from 2006 to 2013. In 2013, the average income per household for the metropolitan group was about $\$ 43,310$, while that of the rural one was about $\$ 38,350$ and they were significantly different at the $5 \%$ level for the two-tailed test $(\mathrm{P}=0.014)$. The rates of obesity and the seven other indicator variables in the two groups, based on aggregated data from 2006 to 2013, were compared via two-sample t-tests. Table 2 shows the results of these comparisons. The rates for obesity, smoking and physical activity for both groups were significantly different at the $5 \%$ level for the twotailed test $(\mathrm{P}=0.001,0.009$ and 0.046 , respectively). There were no significant statistical differences between the two groups for the remaining variables. However, it should be noted that the levels for all vari- ables except depression and physical activity were (slightly) higher in the rural areas, even though the difference was not always statistically significant.

Figure 3 displays the temporal development in the prevalence of obesity for both genders in the regional subgroups. The tests were conducted to determine whether there was an increase over time, i.e., whether the slope of a regression line was positive. The $P$ value for the slopes of adolescent males and adolescent females in rural areas were 0.012 and 0.005 , respectively. Therefore, we can conclude that these slopes were greater than zero, which implies an increasing trend over the study period. There was no significant increase of the slopes of adolescent males and adolescent females in the metropolitan areas $(\mathrm{P}=0.304$ and 0.131 , respectively). Adolescent males in the rural areas had the highest absolute increase in the prevalence of obesity, while adolescent males in the metropolitan areas had the lowest increase. It is also noteworthy that adolescent females in the rural areas had a slightly higher obesity prevalence than adolescent females in the metropolitan areas from 2007 to 2013. For adolescent males, this was only the case for 2012 and 2013 and it was the other way around from 2006 to 2009 .

\section{Visualising data at the local level}

The LM plots combine exploratory data analysis capabilities with traditional statistical graphics while maintaining the geographical con-

Table 2. Comparison of mean by group (\%).

\begin{tabular}{lccc} 
Variable & $\begin{array}{c}\text { Group A } \\
\text { (metropolitan area) }\end{array}$ & $\begin{array}{c}\text { Group B } \\
\text { (rural area) }\end{array}$ & P \\
Obesity & 8.7 & 9.0 & $0.001^{*}$ \\
Physical activity & 10.8 & 10.5 & $0.046^{*}$ \\
\hline Being without breakfast & 25.4 & 26.3 & 0.678 \\
Fast-food consumption & 17.5 & 17.8 & 0.992 \\
\hline Stress & 43.2 & 43.6 & 0.716 \\
Depression & 36.2 & 35.9 & 0.950 \\
\hline Smoking & 11.7 & 13.0 & $0.009^{*}$ \\
Drinking & 21.8 & 22.8 & 0.480 \\
\hline
\end{tabular}

*Statistically significant result at the $5 \%$ level of significance.

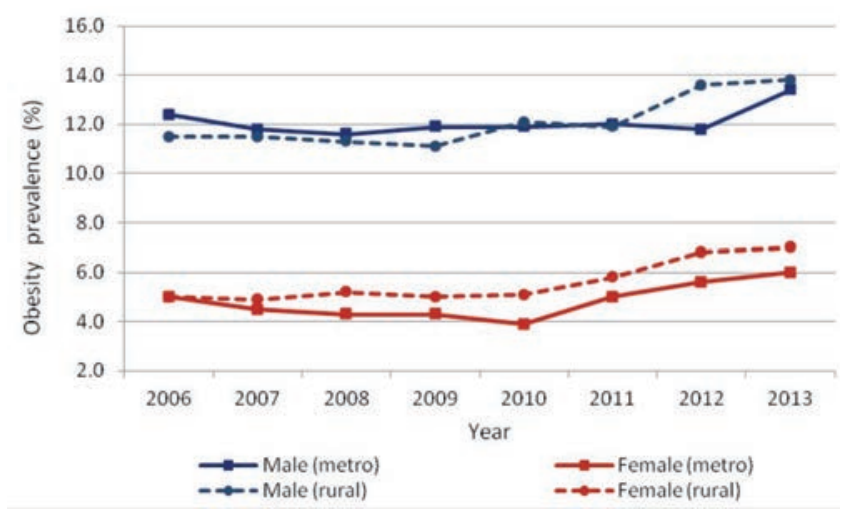

Figure 3. Trends of the prevalence of obesity by year, gender and regional subgroup. 
text of the data. Figure 4 shows a LM plot used to explore the spatial patterns and relationships among obesity, stress, starting the day without breakfast, and depression at the local level.

The numbers between the statistical panels indicate the rank of a local autonomous entity in the statistical panels to the right. The subregions were sorted top to bottom with respect to decreasing prevalence of obesity. The map panels of the LM plot exhibit the geographic pattern: the highest prevalence of obesity (shown in the statistical panel no. 1) was found in the North and East (with Jeju a spatial outlier); the lowest prevalence was found in the West and in the South.

Obesity did not show any association with stress and being without breakfast (Spearman's rho: -0.122 and 0.024, P: 0.653 and 0.931, respectively) but obesity and depression had a negative association (Spearman's rho: -0.560, P: 0.024). In addition, the data in the statisti- cal panel no. 2 (stress) and no. 3 (being without breakfast) as well as in the statistical panel no. 2 (stress) and no. 4 (depression) are highly correlated. In general, stress, eating behaviour and depression have been recognised as important determinants of obesity (Torres and Nowson, 2007; Luppino et al., 2010). However, as shown in Figure 4, obesity in adolescents in Korea were not (or even negatively) associated with these factors. This result complements other studies also based on the KYRBWS data set (Yu, 2011; Byeon, 2013). Many past studies report that people engaged in any of the four factors mentioned in Table 1, i.e. physical activity, smoking, drinking and fast-food consumption, are more likely to also engage in other high-risk behaviour (Strauss, 2000; Brownell, 2004; Baek, 2008; Kleiser et al., 2009; Kries et $a l ., 2014)$, but they had no significant effect in this study (Spearman's rho: $-0.210,-0.186,0.099$, and -0.269 , respectively).

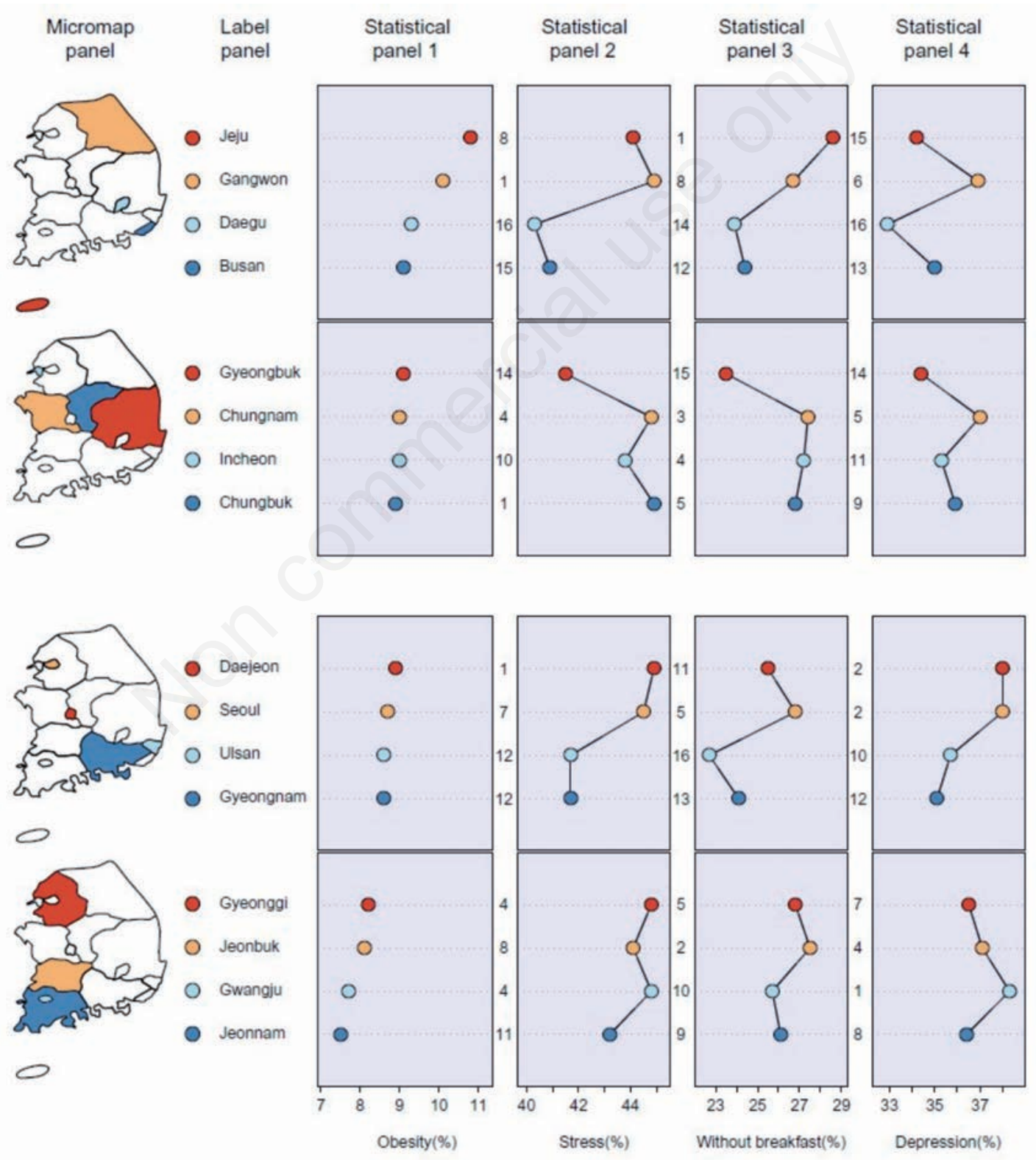

Figure 4. Linked micromap plot showing the relationships among obesity, stress, starting the day without breakfast, and depression based on regional averages in the period 2006-2013. 
Figure 5 shows the regional trends and increases of the prevalence of obesity for the period from 2006 to 2013. Jeju had the largest margin in both absolute increase and relative increase from 2006 to 2013. The relative increases were higher in rural areas than in most metropolitan areas. In 2012, the rates in Gangwon, Jeonbuk and Jeonnam increased considerably but they also decreased considerably again in 2013 , which could simply be due to a data-collection problem in these three subregions in 2012. The obesity rates in 2013, compared to 2006, had increased (slightly) in all regions except in Seoul. Moreover, as a result of the regression analysis, the slopes for seven of the regions (Gwangju, Gyeongnam, Jeonbuk, Jeonnam, Chungnam, Gangwon, and Busan) were significantly greater than zero at the $5 \%$ level for the onetailed test.
Table 3. Comparison of mean by gender (\%).

\begin{tabular}{lcc} 
Variable & Male & Female \\
Physical activity & 15.3 & 6.0 \\
Being without breakfast & 26.0 & 26.1 \\
\hline Fast-food consumption & 19.0 & 16.1 \\
Stress & 34.8 & 49.6 \\
\hline Depression & 31.4 & 41.8 \\
Smoking & 16.5 & 7.2 \\
\hline Drinking & 24.9 & 19.6 \\
\hline
\end{tabular}

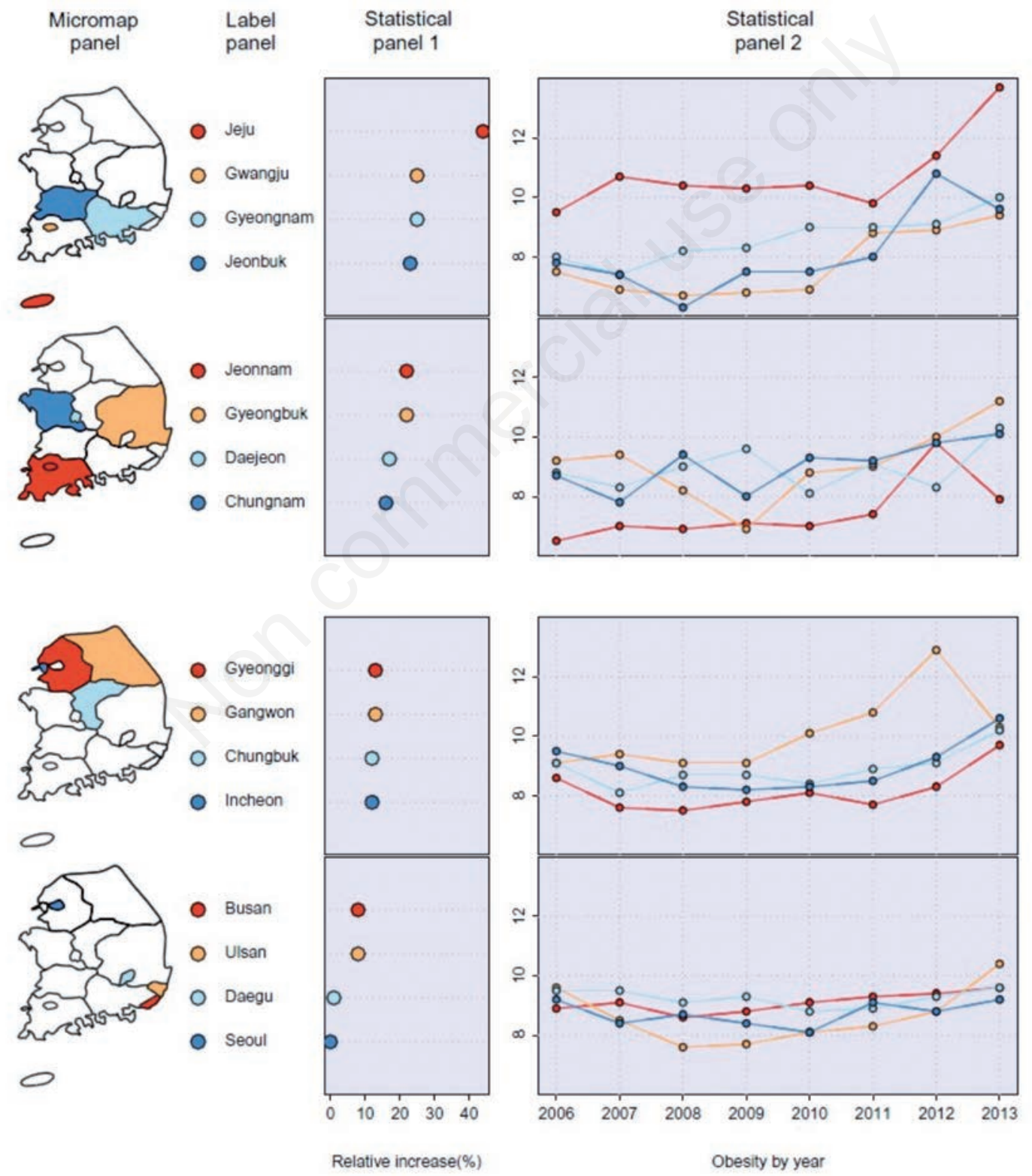

Figure 5. Trends of the prevalence of obesity for the period 2006-2013. 


\section{Discussion}

Obesity in children and adolescents is a major concern, not only because of health and social problems in the short-term, but also because of a high risk that may continue into adulthood and affect long-term health. Over the past two decades, however, the prevalence of obesity is increasing worldwide although the proportion varies from country to country and between geographical areas within a country (Mohsin et al., 2012). In past studies, impoverished areas in developed countries often were associated with higher rates of obesity. For example, in an obesity study for the United States, Ogden et al. (2010) reported that children and adolescents from low income families are more likely to be obese than children and adolescents from higher income families. Therefore, the effects of rural areas and lower income on obesity were confounded in our study.

In this study, we examined the trends of adolescent obesity for the period from 2006 to 2013 in Korea and described how spatial patterns of obesity and other variables can be examined visually and simultaneously using LM plots. First, we investigated the temporal variation of the prevalence of obesity at the national level. Second, we divided the 16 local autonomies of Korea into two groups (metropolitan and rural areas) and compared the variables for these two groups. Finally, we explored the geographic patterns and the relationship between the variables using LM plots.

In summary, the prevalence of obesity of adolescent males was about twice as high, compared to that of adolescent females (Figure 1). Thus, the prevalence of obesity of adolescents in Korea can be said to be determined by gender. Based on this observation, the differences in factors relevant to adolescent obesity (Table 3), derived from the KOSIS data, can be understood as follows: compared with adolescent females, adolescent males are physically more active, smoke and drink more frequently; have less stress and depression; and are approximately equal with respect to eating fast-food and starting the day without breakfast.

The obesity rates for 2013 , compared to 2006 , have increased slightly for Korean adolescents in general. Adolescent males living in rural areas had the highest absolute increase in the prevalence of obesity, while adolescent males living in metropolitan areas had the lowest increase. In addition, the obesity rates for 2013, compared to 2006, increased in all regions except in Seoul. The trends at the local level show a slight increase in all regions of Korea. The regions with high obesity prevalence are all located in the Northeast. The results of this study should facilitate the understanding of the variety of geographically referenced data and visualise more enriched statistical information.

\section{Conclusions}

It should be noted that the data used in this study already had been aggregated. Therefore, it was not possible for us to adjust for covariates such as age and gender in our analysis at the local level. Such an adjustment might eventually help to better explain some of the unexpected patterns observed in our study. In addition, the body mass index used in this study was calculated from self-reported weight and height and not from anthropometric measures. The self-reported measures could lead to a considerable underestimation of the obesity prevalence rates. Moreover, we are describing ecological correlations, i.e., correlations based on group means, rather than on the unaggregated data. On the one hand, this considerably reduces the sample size, e.g. to $\mathrm{n}=8$ when comparing trends from 2006 to 2013. On the other, this also reduces the variation, compared to using the unaggregated data from several thousand participants of the KYRBWS study each year. Therefore, some of the trends we observed, which ultimately were not statistically significant, may become significant when working with the full-unaggregated data set - and vice versa.

\section{References}

Ahn JY, 2013. Visualizing statistical information using Korean linked micromap plots. In: Cho SH, ed. Proceedings of IASC Satellite Conference for the 59th ISI WSC \& The 8th Conference of IASCARS, Asian Regional Section of the IASC, pp 219-221.

Baek S, 2008. Do obese children exhibit distinguishable behaviours from normal weight children - based on literature review. Nutr Res Pract 13:386-95.

Brownell KD, 2004. Fast food and obesity in children. Pediatrics 113:132.

Byeon H, 2013. Relationship between self-perception of weight and depression experience in Korean adolescents. Adv Sci Tech Lett 40:66-9.

Carr DB, 2001. Designing linked micromap plots for states with many counties. Stat Med 20:1331-9.

Carr DB, Pickle LW, 2010. Visualizing data patterns with micromaps. Chapman \& Hall/CRC: Boca Raton, FL, USA.

Carr DB, Pierson SM, 1996. Emphasizing statistical summaries and showing spatial context with micromaps. Stat Comp Graph News 7:16-23.

Dent BD, 1993. Cartography: thematic map design. William C. Brown, Dubuque, IA, USA.

Edsall R, 2003. Design and usability of an enhanced geographic information system for exploration of multivariate health statistics. Prof Geogr 55:605-19.

Ezzati M, Martin H, Skjold S, Hoorn SV, Murray C, 2006. Trends in national and state-level obesity in the USA after correction for selfreport bias: analysis of health surveys. J Roy Soc Med 99:250-7.

Fontaine KR, Redden DT, Wang C, Westfall A0, Allison DB, 2003. Years of life lost due to obesity. J Am Med Assoc 289:187-93.

Gebreab S, Gillies RR, Munger RG, Symanzik J, 2008. Visualization and interpretation of birth defects data using linked micromap plots. Birth Defects Res A 82:110-9.

Han JC, Lawlor DA, Kimm SY, 2010. Childhood obesity. Lancet 375:1737-48.

Han KS, Park SJ, Mun GS, Choi SH, Symanzik J, Gebreab S, Ahn JY, 2014. Linked micromaps for the visualization of geographically referenced data. ICIC Expr Lett 8:443-8.

Harris RL, 1999. Information graphics. A comprehensive illustrated reference. Oxford University Press, New York, NY, USA.

Jacob BG, Krapp F, Ponce F, Gottuzzo E, Griffith DA, Novak RJ, 2010. Accounting for autocorrelation in multi-drug resistant tuberculosis predictors using a set of parsimonious orthogonal eigenvectors aggregated in geographic space. Geospat Health 4:201-17.

James WPT, Jackson-Leach R, Mhurchu CN, Kalamara E, Shayeghi M, Rigby NJ, Nishida C, Rodgers A, 2008. Overweight and obesity (high body mass index). In: Ezzati M, Lopez A, Rodgers A, Murray C, eds. Comparative quantification of health risks: Global and regional burden of disease attributable to selected major risk factors. World Health Organization, Geneva, Switzerland, pp 497-596.

Kleiser C, Rosario AS, Mensink G, Langenohl RP, Kurth BM, 2009. Potential determinants of obesity among children and adolescents 
in Germany: results from the cross-sectional KiGGS study. BMC Publ Health 9:46.

Kolotkin RL, Meter K, Williams GR, 2001. Quality of life and obesity. Obes Rev 2:219-29.

Kries R, Müller MJ, Heinrich J, 2014. Early prevention of childhood obesity: another promise or a reliable path for battling childhood obesity? Obes Facts 7:77-81.

Luppino FS, Wit LM, Bouvy PF, Stijnen T, Cuijpers P, Penninx BW, Zitman FG, 2010. Overweight, obesity, and depression: a systematic review and meta-analysis of longitudinal studies. Arch Gen Psych 67:220-9.

McLaren L, 2007. Socioeconomic status and obesity. Epidemiol Rev 29:29-48.

Mohsin F, Begum T, Azad K, Nahar N, 2012. An overview of childhood obesity. Birdem Med J 2:93-8.

Ogden CL, Lamb MM, Carroll MD, Flegal KM, 2010. Obesity and socioeconomic status in children and adolescents: United States, 20052008. National Center for Health Statistics, Hyattsville, MD, USA.

Pichainarong N, Mongkalangoon N, Kalayanarooj S, Chaveepojnkamjorn W, 2006. Relationship between body size and severity of dengue hemorrhagic fever among children aged 0-14 years. Southeast Asian J Trop Med Public Health 37:283-8.
Puhl RM, Heuer C, 2009. The stigma of obesity: a review and update. Obesity 17:941-64.

Reilly JJ, Wilson 0,2006 . ABC of obesity: childhood obesity. Brit Med J 333:1207-10.

Snedecor GW, Cochran WG, 1989. Statistical methods. Iowa State University Press, Iowa City, IA, USA.

Strauss R, 2000. Childhood obesity and self-esteem. Pediatrics 105:e15.

Symanzik J, Carr DB, 2008. Interactive linked micromap plots for the display of geographically referenced statistical data. In: Chen C, Härdle W, Unwin A, eds. Handbook of data visualization. Springer, Heidelberg, Germany, pp 267-94.

Torres SJ, Nowson CA, 2007. Relationship between stress, eating behavior, and obesity. Nutrition 23:887-94.

Wang X, Chen JX, Carr DB, Bell BS, Pickle LW, 2002. Geographic statistics visualization: web-based linked micromap plots. Comput Sci Eng 4:90-4.

Wisniewski A, Chernausek S, 2009. Gender in childhood obesity: family environment, hormones, and genes. Gender Med 6:76-85.

Yu NS, 2011. A study on perceived weight, eating habits, and unhealthy weight control behavior in Korean adolescents. Int $\mathrm{J}$ Hum Ecol 12:13-24. 